\title{
Extracellular Vesicles Modulate Formation of Transmissive Mutant Huntingtin Assemblies
}

Kyle Nunn ${ }^{1}$, Xuyuan Kuang ${ }^{2}$, Paul Castellano ${ }^{1}$, Jennifer Jiang ${ }^{1}$, Arianna Horgan ${ }^{3}$, Joyce Kong ${ }^{4}$, Zhiqun Tan $^{5}$ and Wei Dai ${ }^{1}$

${ }^{1}$ Department of Cell Biology and Neuroscience, Rutgers, The State University of New Jersey, Piscataway, NJ, USA; Institute for Quantitative Biomedicine, Rutgers, The State University of New Jersey, Piscataway, NJ, USA, United States, ${ }^{2}$ Department of Hyperbaric Medicine, Xiangya Hospital, Central South University, Changsha 10008, China, United States, ${ }^{3}$ School of Environmental and Biological Sciences, Rutgers, The State University of New Jersey, Piscataway, NJ 08854 USA, United States, ${ }^{4}$ Ernest Mario School of Pharmacy,Rutgers, The State University of New Jersey, Piscataway, NJ 08854 USA, United States, ${ }^{5}$ Department of Anatomy and Neurobiology, University of California Irvine School of Medicine, Irvine, CA, USA; Institute for Memory Impairment and Neurological Disorders, University of California-Irvine, Irvine, CA, USA, United States

Huntington's Disease (HD) is an autosomal dominant neurodegenerative disorder that affects the central nervous system, primarily striatal neurons. Onset of the disease typically occurs between the ages of 3050 and is attributed to an expansion of the polyglutamine (polyQ) region in the first exon of the huntingtin (HTT) protein, leading to the presence of mutant HTT (mHTT) aggregates. Additionally, larger expansions of the polyQ region of mHTT are increasingly characteristic of intrinsically disordered proteins (IDPs) in aqueous environments, and results in a lack of fixed 3D structures [1]. Historically however, focus on mHTT has turned towards intracellular components and mechanisms of the toxic protein [2-4]. The ability of mHTT to transmit from cell-to-cell is a well-established phenomenon, recently being shown to have the propensity to exit affected cells and spread to and propagate in neighboring cells, following a potential combination of hypothesized pathways [5-7]. This highlights the importance of exploring extracellular factors and mechanisms that may modulate mHTT transmission.

In this report, we generated a conditioned medium using the 14A2.6 PC-12 cell line [8,9] as a model system for investigating mHTT transmission. Following a 24-hour induction under ponasterone A, we observed extracellular microscopic mHTTex1Q97-EGFP densities (Figure 1b). To further investigate the 3D structure of the extracellular mHTTex1Q97-EFGP densities, the collected medium was 
concentrated and subjected to correlative light and electron microscopy (CLEM) and cryo-electron tomography (cryo-ET). We targeted mHTTex1Q97-EGFP+ densities by correlation of the fluorescent CLEM atlas to cryo-TEM. Tomograms collected revealed that these mHTTex1Q97-EGFP granules consisted of a meshwork of densities surrounded by extracellular vesicles (EVs) (Figure 1c-e). Quantitative Western analysis showed that the concentration of mHTTex1Q97-EGFP protein in the conditioned medium is in the nanomolar range, which is usually too low for pure peptides with extended polyQ tract to spontaneously form large assemblies. Interestingly, the co-localization of EVs and assemblies of mHTTex1Q97-EGFP meshwork suggests that EVs may modulate and promote the formation of the uncoated transmissive mHTT assemblies.

To test this hypothesis, an in vitro reconstitution experiment was designed using synthetic polyQ oligomers (Q40 peptides lacking both the N17 region and proline rich region of exon1 as well as the EGFP tag) and preformed liposomes of 100 - $300 \mathrm{~nm}$, the typical size range for EVs. Q40 peptide seeds were then incubated and prepared for cryo-ET studies. Tomograms collected revealed that Q40 peptides form discrete oligomeric species (Figure 2a). In the presence of liposomes, the oligomers formed a significantly more condensed density (Figure 2b). Additionally, we observed polyQ40 oligomers interacting in close proximity to, or perhaps even binding to, the liposome membranes (Figure 2b, arrows). This observation implicates that EVs have a propensity to modulate formation of extracellular mHTT assemblies. Notably, only the polyQ region in exon1 of mHTT is required for these large meshwork assemblies to form.

These findings not only reveal that transmissive mHTT forms several amorphous oligomeric meshworks in a conditioned medium model, but also suggest that EVs influences the formation of these aggregate assemblies. We confirmed this hypothesis using an in vitro reconstitution approach.PolyQ40 peptides formed discrete oligomers until incubated with liposomes, resulting in previously observed amorphous 
meshwork assemblies. Future probing into additional extracellular structural and biochemical mechanisms that promote not only this modulation but also in how EVs specifically drive this formation can provide key insights into mHTT transmissive behavior.

This work was supported by the National Science Foundation (MCB-2046180) and Rutgers Busch Biomedical Research Grant to W.D. Tomographic data was collected at Rutgers CryoEM\& Nanoimaging Facility. We thank Jason Kaelber and Emre Firlar for their support in data collection. 


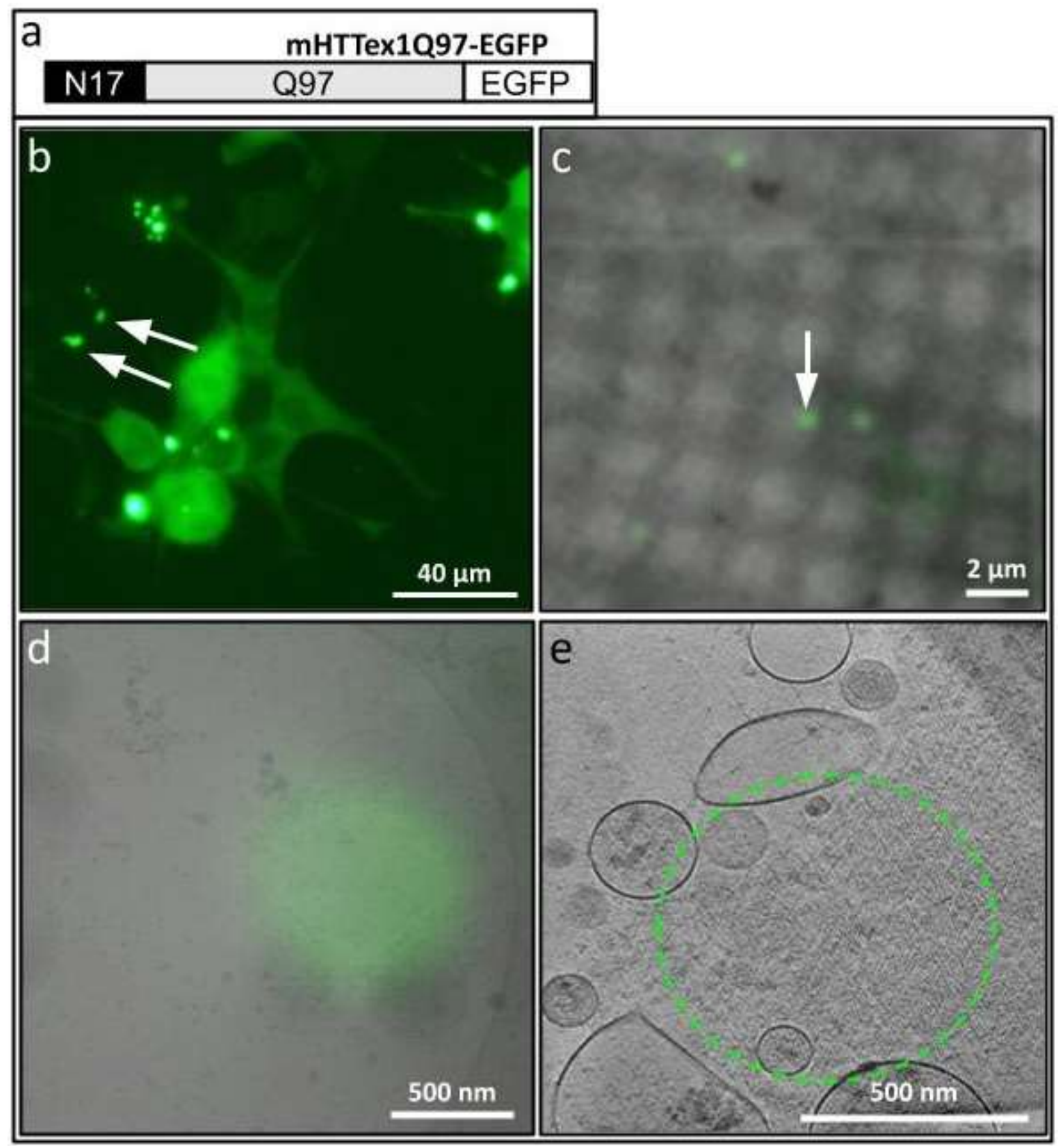

Figure 1. Figure 1. Conditioned medium from 14A2.6 PC-12 cells as a model system for studying extracellular, transmissive mHTT proteins. (a) Schematic diagram of the EGFP labeled, truncated mHTT exon 1 construct (mHTTex1Q97-EGFP). (b) After 24 hour $2 \mu \mathrm{M}$ ponasterone A induction, PC-12 cells secrete transmissive mHTTex1Q97-EGFP granules (arrows). (c) Correlative light microscopy and low magnification electron microscopy (EM) images of a mHTTex1Q97-EGFP granule (arrow). (d) EM image taken at 15,000x magnification with correlative overlay of fluorescence image of mHTTex1-EGFP granule in (c) . (e) Slice view of the mHTTex1Q97-EGFP granule tomogram (green: region of EGFP+ signal). 


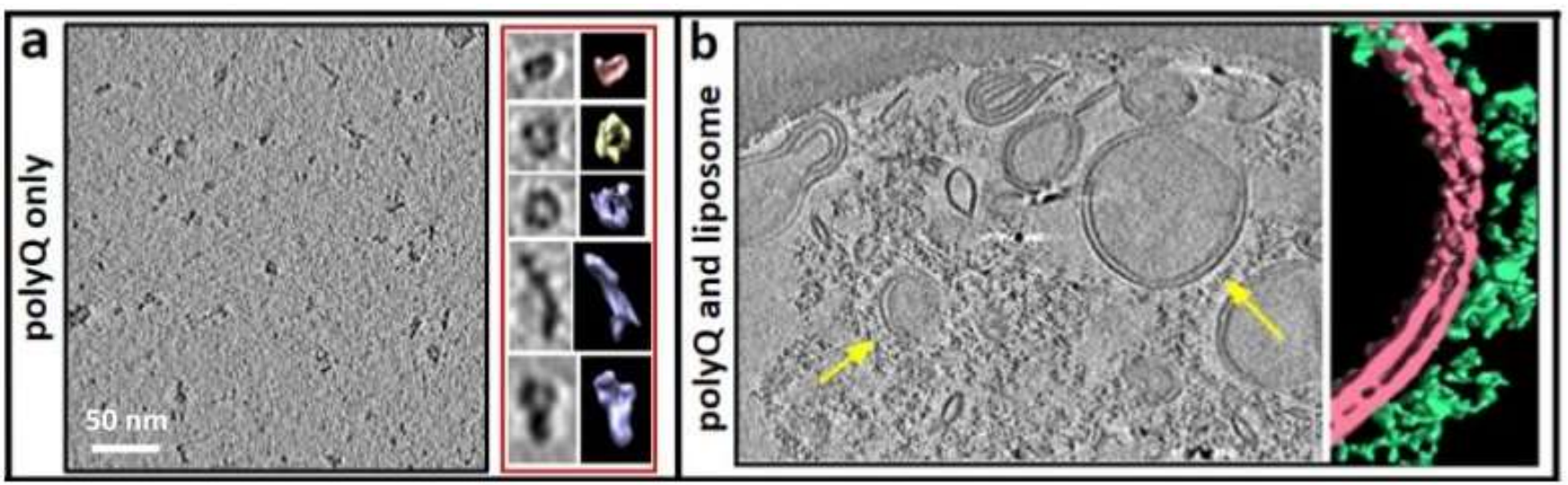

Figure 2. Figure 2. Liposomes modulate synthetic polyQ40 assemblies in vitro. (a) Slice views of discrete polyQ40 oligomers after incubation overnight at room temperature (left) and slice and 3D isosurface views of representative species (right). (b) Slice view of preformed liposome and polyQ40 incubation (left) and representative 3D isosurface view of polyQ40 directly interacting with liposome membrane (right).

References

[1] SL Crick et al, Proc Natl Acad Sci 103 (2006) p.16764

[2] A Gruber et al, Proc Natl Acad Sci 115 (2018) p.E3446

[3] FJB Bäuerleinet al, Cell 171 (2017) p.179

[4] TR Peskettet al, Mol Cell 70 (2018) p.588

[5] M Masnataet al, Front Neurosci11 (2017) p.647

[6] K Trajkovicet al, Bio Protoc8 (2018)

[7] I Jeon et al, Acta Neuropathol132 (2016) p.577

[8] BL Apostol et al, Proc Natl Acad Sci 100 (2003) p.5950

[9] Z Tan et al, Mol Psychiatry 20 (2015) p.1286 